\title{
OBTENCIÓN DE HÍBRIDOS INTERGENÉRICOS HELIANTHUS ANNUUS X TITHONIA ROTUNDIFOLIA Y SU ANÁLISIS MORFOLÓGICO Y MOLECULAR
}

\author{
Miriam Paulina Luévanos-Escareño ${ }^{1}$, M. Humberto Reyes-Valdés ${ }^{*}$, José Ángel \\ Villarreal-Quintanilla ${ }^{2}$ y RaÚl RodRíGUEZ-HerRera ${ }^{3}$ \\ ${ }^{1}$ Universidad Autónoma Agraria Antonio Narro, Departamento de \\ Fitomejoramiento, Calzada Antonio Narro 1923, \\ Colonia Buenavista, 25315 Saltillo, Coahuila, México. \\ ${ }^{2}$ Universidad Autónoma Agraria Antonio Narro, \\ Departamento de Botánica, Calzada Antonio Narro 1923, \\ Colonia Buenavista, 25315, Saltillo, Coahuila, México. \\ ${ }^{3}$ Universidad Autónoma de Coahuila, Facultad de Ciencias Químicas, \\ Boulevard. Venustiano Carranza e Ing. José Cárdenas s/n, \\ Colonia República, 25280 Saltillo, Coahuila, México.
}

\section{RESUMEN}

Se realizó el cruzamiento entre el girasol cultivado Helianthus annuus y la especie silvestre con potencial ornamental Tithonia rotundifolia. De tres materiales cultivados: AN-3, Primavera y HA 89, sólo se tuvo éxito con la línea pública de girasol cultivado HA 89 como progenitor femenino. A su vez el masculino fue una población de T. rotundifolia recolectada en el estado de Guerrero, México. Se produjeron 826 aquenios híbridos bien desarrollados, con una tasa de éxito de cuatro en 1000 aquenios potenciales. Dentro de una muestra de 49 plantas establecidas en el campo experimental, se observaron dos clases fenotípicas: a) individuos con muchas inflorescencias pequeñas $y$ b) otros con cabezuela solitaria grande y con pocas o ninguna inflorescencia axilar. Ambos tipos fueron caracterizados morfológicamente y presentaron rasgos claramente híbridos. Además, se analizó la huella genética, a través del polimorfismo en la longitud de los fragmentos amplificados (AFLPs), de una muestra de diez tríos familiares de cruzamientos, con un promedio de 28 polimorfismos por trío. El análisis de huella genética de las plantas complementó al estudio morfológico, permitió

\footnotetext{
*Autor para la correspondencia:mhreyes@uaaan.mx, mathgenome@gmail.com
} 
constatar la naturaleza híbrida de las progenies y descartar el fenómeno del cruzamiento parcial. Todas las plantas experimentales obtenidas resultaron ser estériles. Del presente trabajo se desprende que se requiere de manipulación cromosómica o técnicas de cultivo de tejidos para el desarrollo de híbridos fértiles con potencial ornamental.

Palabras clave: AFLP, esterilidad, Helianthus annuиs, híbridos intergenéricos ornamental, Tithonia rotundifolia.

\begin{abstract}
Hybridization between the cultivated sunflower Helianthus annuus and the wild species with ornamental potential Tithonia rotundifolia was performed. From three cultivated materials: AN-3, Primavera and HA 89, the only success was obtained with the public cultivated sunflower line HA 89 as female parent. The male parent was a T. rotundifolia population collected in the state of Guerrero, Mexico. A total of 826 well developed hybrid achenes were produced, with a rate of success of four in 1000 potential achenes. In a sample of 49 plants established in the experimental field, two phenotypic classes were observed: a) plants with many small inflorescenses, and b) plants with a single big head and a few or none axillary inflorescenses. Both types were morphologically characterized and showed a clearly hybrid morphology. Additionally, the AFLP-based DNA fingerprints were analyzed in a sample of ten familial trios, with an average of 28 polymorphisms per trio. The DNA fingerprinting analysis of the plants complemented the morphological study, allowed confirmation of the hybrid nature of the progenies and ruled out the partial hybridization phenomenon. All the hybrid plants showed sterility. From this work, it becomes clear that chromosomic manipulation or tissue culture techniques are needed to develop fertile hybrids with ornamental potential.
\end{abstract}

Key words: AFLP, Helianthus annuus, intergeneric hybrids, ornamental, sterility and Tithonia rotundifolia.

\title{
INTRODUCCIÓN
}

El género Helianthus pertenece a la tribu Heliantheae dentro de la familia Asteraceae. Consta de 49 especies, de las cuales 13 son anuales y 36 perennes (Schilling y Heiser, 1981), con un número básico de cromosomas x $=17$ (Heiser, 1978). El girasol cultivado (Helianthus annuиs) es una especie de gran importancia a nivel 
mundial por su alto contenido de aceite; sus semillas son utilizadas en confitería y alimento para animales, y además es bien conocida como planta ornamental (Robles, 1986; Alba y Llanos, 1990).

Tithonia rotundifolia (Upfold y Van Staden, 1990) es una planta silvestre, relacionada con Helianthus, distribuida en México y registrada como nativa de América Central. Su número cromosómico es igual al de $H$. annuus $(2 \mathrm{n}=34)$ y ambas pertenecen a la familia Asteraceae. Esta especie ha sido utilizada como ornamental en países como Estados Unidos de América, y existen variedades domesticadas comerciales de la misma. Su potencial ornamental está basado en el atractivo color naranja de sus inflorescencias así como en su pubescencia suave, que contrasta con aquella híspida de $H$. annuus.

Hay solamente dos reportes de hibridación entre Tithonia y Helianthus en la literatura. El primero de ellos (Cristov y Panayotov, 1991) consistió en la realización de cruzamientos entre $H$. annuus cultivado y $T$. rotundifolia. Los materiales domesticados de $H$. annuus, usados como progenitor femenino, fueron la variedad Peredovik, y las líneas 3004 y HA 89. Los cruzamientos de T. rotundifolia con Peredovik y la línea 3004 dieron como resultado plantas con una sola inflorescencia, mientras que del cruzamiento de HA 89 con $T$. rotundifolia se obtuvieron individuos muy ramificados. De acuerdo con los autores, este último carácter se debe a la interacción entre los genes de capítulos múltiples de T. rotundifolia, y los alelos recesivos en la línea HA 89, procedentes de $H$. argophyllus durante la incorporación de la esterilidad masculina. Las plantas híbridas fueron obtenidas solamente cuando los progenitores cultivados se usaron como hembras. Se reporta que los individuos obtenidos por cruzamiento fueron fértiles tanto para autopolinización como para polinización cruzada. En dicho trabajo no se hizo un estudio molecular que comprobara la naturaleza híbrida de las plantas.

En el segundo reporte de hibridación (Reyes-Valdés et al., 2005), el progenitor femenino fue la línea pública de $H$. annuus HA 89 y el masculino una planta de $T$. rotundifolia de origen silvestre del estado de Guerrero, México. Se logró obtener un descendiente híbrido de cabezuela solitaria, estéril y con características morfológicas de ambos progenitores. Se demostró su naturaleza híbrida por medio de análisis de AFLPs (polimorfismo en la longitud de fragmentos amplificados) y se descartó el fenómeno de hibridación parcial. La obtención de un híbrido con cabezuela solitaria contrasta con lo reportado por Cristov y Panayotov (1991). Sin embargo, la versión androestéril de la línea HA 89 utilizada por Reyes-Valdés et al., (2005) no tiene citoplasma procedente de $H$. argophyllus, sino que posee el clásico francés CMS PET1 de H. petiolaris (Leclercq, 1969). 
Con la finalidad contribuir al conocimiento del comportamiento genómico en asteráceas y abrir la posibilidad de desarrollar una nueva planta ornamental, así como permitir la transferencia bidireccional de genes entre $H$. annuus y $T$. rotundifolia, en este trabajo se hizo una labor de cruzamiento intensivo entre los materiales originalmente utilizados por Reyes-Valdés et al., (2005). Los objetivos particulares consistieron en obtener una muestra suficientemente amplia de híbridos intergenéricos, caracterizarlos morfológicamente y validar su naturaleza cruzada con huella genética a través de la técnica de AFLPs (Vos et al., 1995).

\section{MATERIALES Y MÉTODOS}

Cruzamientos

El material genético para la formación de híbridos intergenéricos estuvo conformado por la línea androestéril CMS HA 89 de girasol cultivado (H. annuus) como progenitor femenino. La especie silvestre T. rotundifolia procedente de una población de Iguala, Guerrero y con clave de colecta Ac26, fue utilizada como progenitor masculino. En ensayos preliminares se usaron también los materiales de girasol cultivado AN-3 y Primavera, sin embargo no se tuvo éxito en los cruzamientos, por lo cual fue únicamente la línea HA 89 en su versión androestéril CMS HA 89 la que se utilizó intensivamente en los cruzamientos, y de la cual se polinizaron 100 capítulos.

Se realizó la siembra de semillas del girasol cultivado H. annuus (CMS HA 89) y de $T$. rotundifolia (Ac26) en condiciones de vivero. Se transplantaron los materiales al campo experimental Buenavista, en Saltillo, Coahuila, para realizar los cruzamientos. Se recolectó polen fresco de T. rotundifolia en cajas de Petri para fertilizar los capítulos de manera artificial. La polinización se realizó con un aplicador de franela dos veces, con intervalo de dos días para asegurar la cruza.

Al término de la madurez fisiológica de CMS HA 89, se cosecharon los aquenios y se determinó el porcentaje de semillas completamente desarrolladas por cada cruzamiento, a través de la cuenta de aquenios llenos (desarrollados) y los vacíos (no desarrollados). Después se sumó el total de semillas por cada planta, se calculó el porcentaje individual, y posteriormente se estimó un porcentaje promedio por cruzamiento. En los cruzamientos se empleó la siguiente identificación: CMSHA89 denota a la línea progenitora de $H$. annuus, con plantas marcadas numéricamente. TR es la especie T. rotundifolia Ac26, con individuos reconocidos por las letras A, 
C, D, F, G, I, H y K. No se anotan las claves de los machos que no tuvieron descendencia.

Evaluación de las plantas híbridas

La progenie de los cruzamientos se estudió en el campo experimental Buenavista. Los híbridos se establecieron y se les hizo un análisis morfológico con el fin de hacer su descripción. Se evaluaron y compararon estadísticamente en cuanto a diámetro de capítulo y altura de planta los dos tipos morfológicos encontrados. La comparación se hizo a través de la prueba " $\mathrm{t}$ " de Student y se utilizó como herramienta analítica el ambiente estadístico $\mathrm{R}$ versión 2.7.0. (R Development Core Team, 2008).

Análisis de huella genética

Para el asilamiento de ADN, se recolectaron las hojas inferiores de las plantas en estado adulto de los progenitores verdaderos correspondientes a la línea CMS HA 89 y a T. rotundifolia, así como de la progenie de los cruzamientos. La técnica de extracción de ADN fue la propuesta por Doyle y Doyle (1990), la cual se modificó con el uso de una varilla de vidrio, doblada en forma de gancho, para separar manualmente ADN de alto peso molecular. En el almacenamiento de hojas vegetales a largo plazo para extracción de ADN, se recurrió a la deshidratación y conservación a temperatura ambiente.

El análisis molecular a través de AFLP (polimorfismo en la longitud de fragmentos amplificados) propuesto por Vos et al. (1995), fue realizado con el protocolo "IRDye Fluorescent AFLP Kit for Large Plant Genome Analysis LI-CoRß (Biosciences) con un software SAGA ${ }^{\mathrm{MX}}$ versión 3.2, que utiliza dos colorantes fluorescentes $(700$ y $800 \mathrm{~nm})$. Esta metodología se basó en cuatro etapas: una primera, en la cual las muestras de ADN fueron digeridas con dos enzimas: una de corte frecuente MseI y otra de corte poco frecuente EcoRI. En la segunda se emplearon adaptadores específicos para pegar los bordes de los fragmentos recién formados y proveer una secuencia conocida, se utilizó la enzima T4 ligasa para facilitar la unión de los extremos de las secuencias conocidas. Para las siguientes etapas se recurrió a la técnica de amplificación PCR (reacción de polimerización en cadena) empleando iniciadores marcados en las secuencias ligadas. En el paso tres se llevó a cabo una pre-amplificación de las muestras utilizando iniciadores de secuencia conocida, esto produjo un conjunto de fragmentos con la secuencia complementaria al iniciador. 
En la etapa cuatro se hizo una amplificación selectiva usando una combinación de los iniciadores EcoRI E-ACA (700) y E-ACT (800), y E-ACC (700) y E-ACT (800), marcados con fluorescencia y con iniciadores M-CAG. Se realizó una electroforesis en gel de poliacrilamida a $6 \%$ en un secuenciador LI-CoR ${ }^{\circledR}$ (Biosciences) para verificar los productos de PCR obtenidos en la amplificación selectiva.

Se analizaron las huellas genéticas en cuanto a bandas compartidas entre progenie y progenitores, así como para la estimación de distancias euclidianas, a través de un programa diseñado ex profeso en $\mathrm{R}$ versión 2.7.0. (R Development Core Team, 2008). La simetría entre las distancias euclidianas progenitor-híbrido constituyen una prueba para descartar la hibridación parcial (Reyes-Valdés et al., 2005).

\section{RESULTADOS Y DISCUSIÓN}

En el Cuadro 1 se presentan las estadísticas de producción de aquenios por cada planta progenitora masculina (T. rotundifolia Ac26). De un total de 100 capítulos polinizados, 47 generaron alguna cantidad de frutos desarrollados, cuyas proporciones con respecto al total se indican en la última columna. El porcentaje promedio de producción de aquenios en estos 47 capítulos fue de 0.93 . Teniendo en cuenta que estos últimos solamente representan $47 \%$ del total, se puede inferir que la formación de frutos en este experimento de cruzamiento intergenérico se reduce a un aproximado de $0.4 \%$. Es decir, sólo se espera obtener cuatro semillas desarrolladas de cada 1000 flósculos potencialmente fértiles. Esto refleja el alto grado de incompatibilidad entre las dos especies, que sin embargo no es sorprendente tratándose de miembros de diferentes géneros. Se obtuvo un total 826 aquenios desarrollados, siendo los cruzamientos CMSHA89*TRA, CMSHA89*TRC y CMSHA89*TRG los mayores productores (Cuadro 1).

Evaluación de semilla híbrida

De 210 aquenios híbridos sembrados en verano de 2007, se lograron establecer hasta la etapa reproductiva un total de 49 plantas, debido a que no todas las semillas germinaron y algunas plántulas presentaron malformaciones y no llegaron a la etapa de madurez. Se observaron dos tipos de individuos: aquellos con inflorescencia de varias cabezuelas pequeñas (Híbrido 1) y los que desarrollaron inflorescencia de una sola cabezuela grande (Híbrido 2). Un total de 19 tuvieron 
Cuadro 1. Semilla obtenida en los distintos cruzamientos realizados entre plantas de Helianthus annuus (CMS HA 89), codificada como CMSHA89 y de Tithonia rotundifolia (Ac26), marcadas como TR. En las genealogías de utiliza el símbolo * para indicar cruzamiento.

\begin{tabular}{lcccc}
\hline $\begin{array}{l}\text { Genealogía de } \\
\text { cruzamiento }\end{array}$ & $\begin{array}{l}\text { Cruzamientos } \\
\text { realizados }\end{array}$ & $\begin{array}{l}\text { Plantas formado- } \\
\text { ras de semilla por } \\
\text { cruzamiento }\end{array}$ & $\begin{array}{l}\text { Total de semillas } \\
\text { desarrolladas }\end{array}$ & $\begin{array}{l}\text { Promedio del } \\
\text { porcentaje de } \\
\text { semillas de- } \\
\text { sarrolladas en } \\
\text { los cruzamien- } \\
\text { tos exitosos }\end{array}$ \\
\hline CMSHA89*TRA & 2 & & 206 & 0.88 \\
CMSHA89*TRC & 23 & 11 & 367 & 3.44 \\
CMSHA89*TRD & 15 & 7 & 29 & 0.54 \\
CMSHA89*TRF & 19 & 6 & 10 & 0.19 \\
CMSHA89*TRG & 11 & 10 & 173 & 1.59 \\
CMSHA89*TRH & 5 & 4 & 7 & 0.13 \\
CMSHA89*TRI & 20 & 5 & 29 & 0.44 \\
CMSHA89*TRK & 5 & 2 & 5 & 0.25 \\
Total & 100 & 47 & 826 & 7.46 \\
Promedio & 12.50 & 5.88 & 103.25 & 0.93 \\
\hline
\end{tabular}

la característica de Híbrido 2, y 30 la de Híbrido 1, lo que nos da proporciones de $35 \%$ - 65\%. Lo anterior contrasta con lo reportado por Cristov y Panayotov (1991), quienes obtuvieron solamente plantas ramificadas con el progenitor HA 89. Cabe aclarar que la línea usada por dichos autores tiene citoplasma androestéril de $H$. argophyllus, mientras que la utilizada en este trabajo lo tiene de $H$. petiolaris. Las diferencias genéticas originadas por la infiltración de distintas especies silvestres pueden ser causales de la discrepancia en la morfología de los híbridos. Por otro lado, las poblaciones de T. rotundifolia empleadas en estos trabajos son distintas, y puede haber una variación en frecuencias alélicas para la ramificación que esté influyendo en los resultados.

Descripción morfológica de los dos tipos de híbridos

Híbrido 1 (Inflorescencia de varias cabezuelas pequeñas). Fig. 1A. Hierba anual de 50 a $70 \mathrm{~cm}$ de alto; tallos ramificados en la base, pilosos; hojas alternas, con pecíolos de 6 a $8 \mathrm{~cm}$ largo, láminas rómbicas, de 5 a $14 \mathrm{~cm}$ de largo y 6 a $10 \mathrm{~cm}$ de 

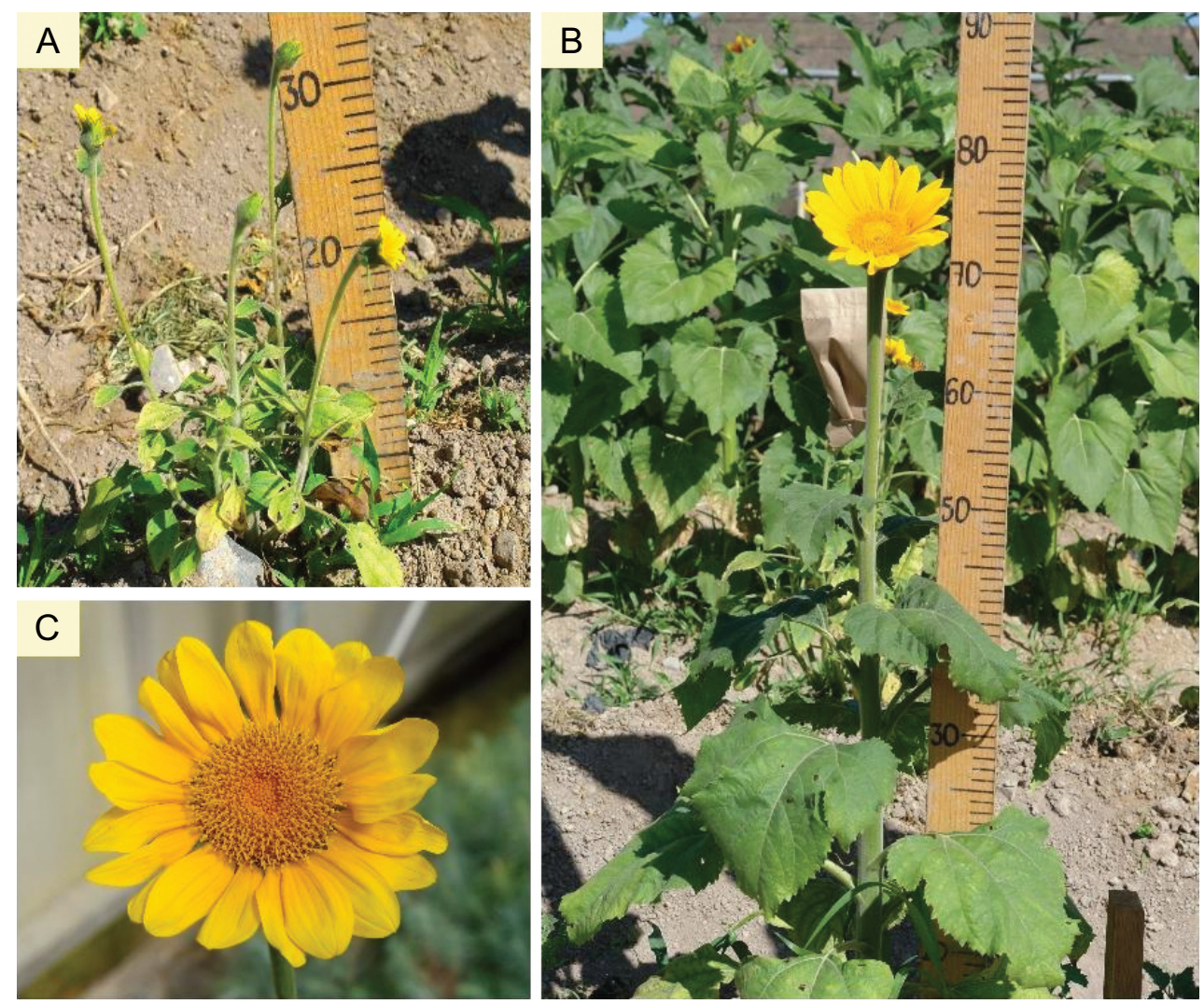

Fig. 1. A. planta híbrida con inflorescencia de varias cabezuelas pequeñas (Híbrido 1); B. planta híbrida con inflorescencia de una cabezuela grande (Híbrido 2); C. detalle de inflorescencia híbrida.

ancho, la base cuneada, el ápice agudo, el borde crenado, pilosas, con pelos ásperos, de color verde amarillento; cabezuelas varias en la parte terminal, de 3 a $4 \mathrm{~cm}$ de diámetro, en pedúnculos fistulosos de 17 a $20 \mathrm{~cm}$ de largo, pilosos, brácteas involucrales en 2 hileras, oblongas, de 1 a $2 \mathrm{~cm}$ de largo y 8 a $10 \mathrm{~mm}$ de ancho, pilosas; receptáculo ligeramente convexo de 2.5 a $3.5 \mathrm{~cm}$ de diámetro; flores liguladas 18 a 20, de 30 a $35 \mathrm{~mm}$ de largo, amarillas; flores del disco 30 a 60, corolas de 5 a $7 \mathrm{~mm}$ de largo, amarillas; aquenios oblongos, base cuneada, de 6 a $9 \mathrm{~mm}$ de largo, 2.5 a $3.5 \mathrm{~mm}$ de ancho, aplanados lateralmente, velutinos; vilano de 2 escamas aristadas subyugales, de 3 a $4.5 \mathrm{~mm}$ de largo y 4(6) escamas ovadas ligeramente laceradas, de 0.5 a $2.0 \mathrm{~mm}$ de largo. 
Híbrido 2 (Inflorescencia de cabezuela solitaria grande). Fig. 1B. Hierba anual de 80 a $110 \mathrm{~cm}$ de alto; tallos no ramificados en la base, pilosos; hojas alternas, con pecíolos de 8 a $10 \mathrm{~cm}$ largo, láminas rómbico-triangulares, de 16 a $20 \mathrm{~cm}$ de largo y 8 a $15 \mathrm{~cm}$ de ancho, la base cuneada, ligeramente hastada, el ápice agudo a obtuso, el borde crenado, pilosas, con pelos ásperos, de color verde oscuro; cabezuelas solitarias en la parte terminal, de 7 a $8 \mathrm{~cm}$ de diámetro, en pedúnculos fistulosos de 18 a $20 \mathrm{~cm}$ de largo, pilosos, brácteas involucrales en 2 hileras, oblongas, de 2 a $4 \mathrm{~cm}$ de largo y 8 a $10 \mathrm{~mm}$ de ancho, pilosas; receptáculo ligeramente convexo de 5 a $7 \mathrm{~cm}$ de diámetro; flores liguladas 18 a 22, de 40 a $60 \mathrm{~mm}$ de largo, amarillas; flores del disco 30 a 60 , corolas de 5 a $7 \mathrm{~mm}$ de largo, amarillas; aquenios oblongos, base cuneada, de 6 a $9 \mathrm{~mm}$ de largo, 2.5 a $3.5 \mathrm{~mm}$ de ancho, aplanados lateralmente, velutinos; vilano de 2 escamas aristadas subyugales, de 3 a $4.5 \mathrm{~mm}$ de largo y 4(6) escamas ovadas ligeramente laceradas, de 0.5 a $2.0 \mathrm{~mm}$ de largo.

Todas las plantas híbridas fueron estériles, ya que no produjeron polen (Fig. 1C) ni semilla. Los resultados presentados en esta etapa son diferentes de los registrados por Cristov y Panayotov (1991) en el estudio de un cruzamiento intergenérico entre $H$. annuиs y $T$. rotundifolia, ya que ellos obtuvieron plantas fértiles. Sin embargo, cabe aclarar que no existe ninguna referencia posterior a un seguimiento de dicho trabajo o a las generaciones subsecuentes de los cruzamientos reportados.

Los valores de medias de altura y diámetro de capítulo para los dos tipos de híbridos se presentan en el Cuadro 2. El del número 2 fue significativamente más

Cuadro 2. Comparación de medias de altura y diámetro de capítulo en plantas híbridas procedentes de los cruzamientos realizados entre Helianthus annuus (CMS HA 89) y Tithonia rotundifolia (Ac 26).

\begin{tabular}{lllcccc}
\hline \multicolumn{1}{c}{ Variable } & Muestra & $\mathrm{N}$ & Media & $\begin{array}{c}\text { Desviación } \\
\text { estándar }\end{array}$ & $\mathrm{t}$ de Student & Valor de P \\
\hline $\begin{array}{l}\text { Altura de planta } \\
(\mathrm{cm})\end{array}$ & Híbrido 1 & 30 & 33.27 & 10.18 & -9.04 & $3.766 \mathrm{e}-09^{* *}$ \\
Diámetro de & Híbrido 2 & 19 & 79.05 & 20.52 & & \\
capítulo $(\mathrm{cm})$ & Híbrido 1 & 30 & 1.375 & 0.98 & -8.17 & $0.0002543^{* *}$ \\
\hline
\end{tabular}

Híbrido 1: inflorescencias de varias cabezuelas pequeñas; Híbrido 2: inflorescencia de cabezuela solitaria grande; N: tamaño de muestra; ** altamente significativo. 
alto y con mayor diámetro de la cabezuela que el del 1. La naturaleza solitaria de esta última le permite ser de tamaño grande, como en los girasoles cultivados, ya que el aporte de nutrientes se canaliza a un capítulo principal. Se observaron casos de capítulos axilares en tales híbridos, pero éstos fueron por lo general muy pequeños y tardíos.

Análisis molecular con AFLPs

Se esperaba que las plantas obtenidas del cruzamiento entre $H$. annuus y $T$. rotundifolia compartieran bandas polimórficas de ambos progenitores. En particular, el híbrido debe exhibir la mayor parte de las bandas únicas de cada uno de los progenitores. Al respecto, los resultados del análisis de huella genética con AFLPs se muestran en el Cuadro 3.

Para los diez tríos familiares muestreados, el progenitor femenino $H$. annuus (CMS HA 89) presentó un promedio de 15.5 bandas únicas, mientras que T. rotundifolia (Ac26) exhibió el valor de 12.25, con un promedio de 28 bandas polimórficas totales. Los híbridos obtenidos compartieron $100 \%$ de las bandas de los progenitores, tanto de $H$. annuus como de $T$. rotundifolia. Tales resultados son consistentes con lo que se esperaría para híbridos verdaderos entre estas dos plantas. Por otro lado, ya que los porcentajes de bandas heredadas son iguales (100\%), se tiene evidencia de simetría en el material genético proveniente de los dos progenitores. Las distancias cuadráticas euclidianas de igual modo, muestran simetría y aditividad, con valores medios de 12.5, 28 y 15.5 para las distancias $H$. annuus híbrido, $H$. annuus - T. rotundifolia y T. rotundifolia - híbrido. En todos los casos el cuadrado de la distancia euclidiana entre los progenitores es igual a las suma de las dos restantes. Estos resultados son también consistentes con la naturaleza híbrida de las progenies. Además, permiten descartar el fenómeno de hibridación parcial reportado por Faure et al. (2002) para cruzas interespecíficas dentro del género Helianthus, manifestada en asimetría genómica en los híbridos. Como puede verse en el Cuadro 3, algunos de los progenitores que fueron utilizados más de una vez en los tríos familiares presentaron diferentes números de bandas. Lo anterior se debe a que al realizar electroforesis independientes, en varios casos no se pudieron visualizar todas. En la Fig. 2 se señalan con flechas algunas bandas polimórficas que, como puede observarse, provienen de uno solo de los progenitores y son compartidas por los híbridos.

Los resultados de huella genética coinciden básicamente con lo reportado por Reyes-Valdés et al., (2005), quienes indican que no hubo evidencia de hibridación 
Luévanos-Escareño et al.: Obtención de híbridos Helianthus annuus x Tithonia rotundifolia

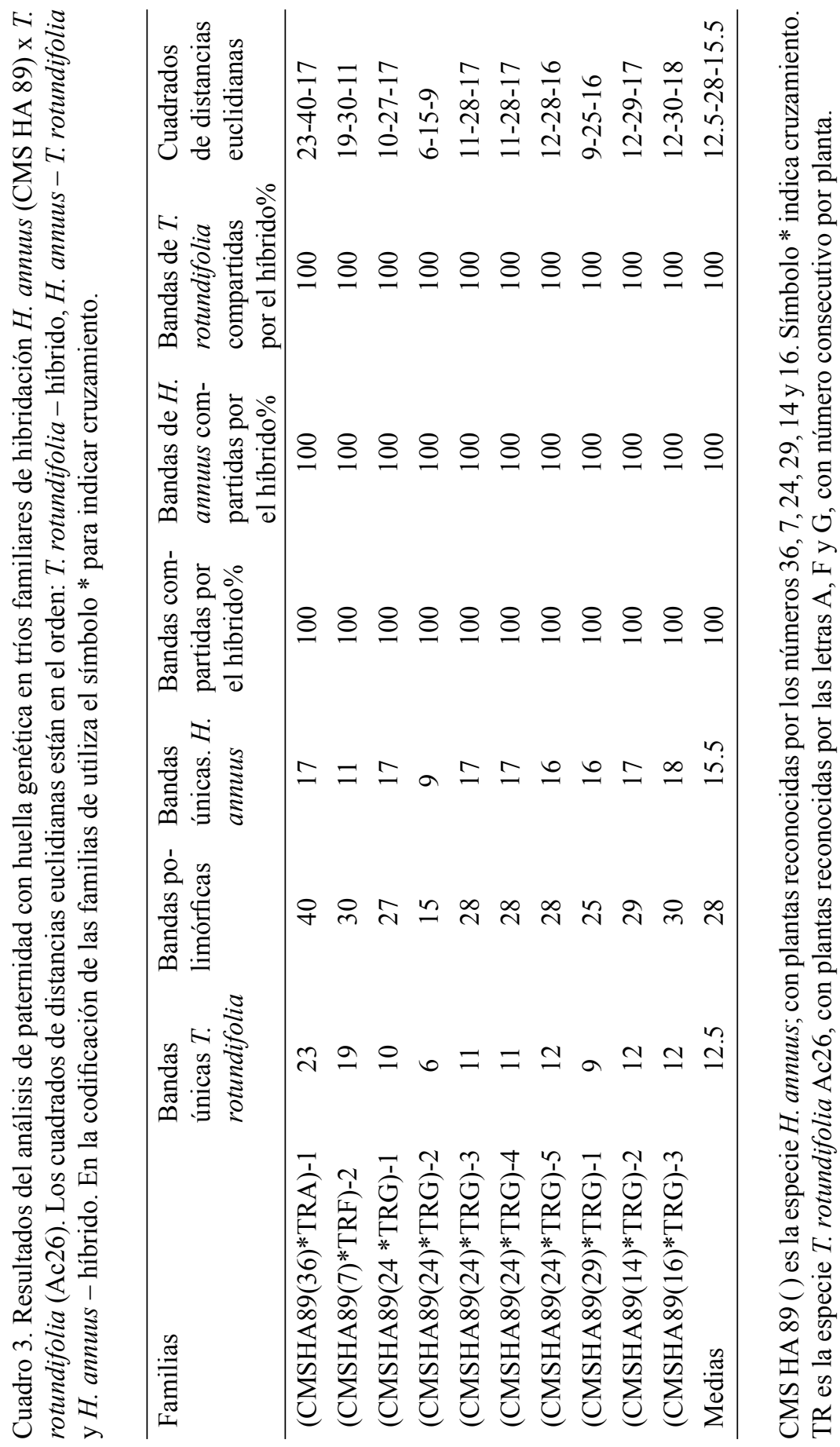




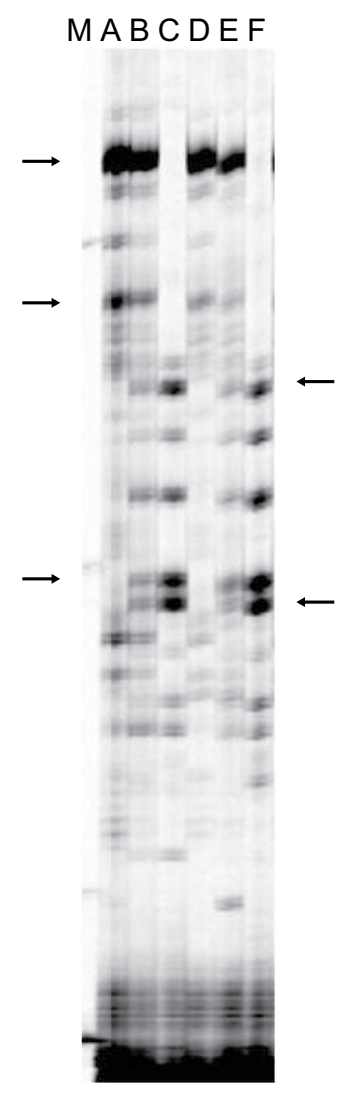

Fig. 2. Huella genética híbridos de girasol. $\mathrm{M}=$ marcador de peso molecular $800 \mathrm{pb}, \mathrm{A}=$ macho T. rotundifolia (TRA), B = híbrido (CMSHA89(36)*TRA); C = hembra CMSHA89(36), D = macho T. rotundifolia (TRG), E = híbrido (CMSHA89(24)*TRG), $\mathrm{F}=$ hembra CMSHA89(24). Las flechas indican bandas polimórficas de alguno de los dos padres, compartidas por el híbrido. El símbolo “*” en los códigos de los híbridos significa cruzamiento.

parcial en los experimentos en los que se utilizaron las mismas especies. Sin embargo, en dicho trabajo no se sujetaron los progenitores verdaderos del híbrido a las pruebas moleculares, a diferencia de esta investigación, en la cual el empleo del $\mathrm{ADN}$ le da mayor precisión al estudio. Por otro lado, en esta contribución se usa una muestra bien representada de 49 híbridos, de los cuales diez fueron analizados por esta vía. 


\section{CONCLUSIONES}

Se logró la obtención de híbridos intergénericos del cruzamiento de Helianthus annuus x Tithonia rotundifolia. Los análisis morfológicos y moleculares con AFLPs, demostraron la naturaleza híbrida de los individuos. El estudio molecular de la progenie indicó que existe simetría en la representación de los genomas parentales, lo cual descarta el fenómeno de hibridación parcial. Todas las plantas híbridas fueron estériles: no produjeron polen, y fueron incapaces de formar semilla. Todo esto indica que aunque las dos especies tienen la suficiente compatibilidad para cruzarse, su divergencia genómica es capaz de causar esterilidad: tal barrera reproductiva, en todo caso podría ser superada a través de manipulación en el laboratorio.

\section{AGRADECIMIENTOS}

Este trabajo de investigación fue financiado por la Secretaría de Agricultura, Ganadería, Desarrollo Rural, Pesca y Alimentación, por el Consejo Nacional de Ciencia y Tecnología (Proyecto SAGARPA-CONACYT con clave 11971) y por la Universidad Autónoma Agraria Antonio Narro.

\section{LITERATURA CITADA}

Alba, A. y M. Llanos 1990. El cultivo del girasol. Ediciones Mundiprensa. Madrid. pp 1316.

Cristov, M. y L. Panayotov. 1991. Hybrids between the genera Helianthus and Tithonia and their study. Helia 14: 27-34.

Doyle, J. J. y J. L. Doyle. 1990. Isolation of plant DNA from fresh tissue. Focus 12: 13-15.

Faure, N., H. Serieys, E. Cazaux, F. Kaan y A. Bervillé. 2002. Partial hybridization in wide crosses between cultivated sunflowers and the perennial Helianthus species H. mollis and H. orgyalis. Ann. Bot. 89: 31-39.

Heiser, C. B. Jr. 1978. Taxonomy of Helianthus and origin of domesticated sunflower. In: Carter, J. F. (ed.). Sunflower science and technology, Agronomy Series 19, American Society of Agronomy, Inc. Madison. pp. 31-35.

Leclercq, P. 1969. Une stérilité male cytoplasmique chez le tournesol. Ann. Amelior. Plantes 19: 99-106.

R Development Core Team. 2008. R: A language and environment for statistical computing, R Foundation for Statistical Computing, Viena Austria. ISBN 3-900051-07-0, URL. http://www.R-Project.org. 
Reyes-Valdés, M. H., M. Gómez-Martínez, O. Martínez y F. Hernández. 2005. Intergeneric hybrid between cultivated sunflower (Helianthus annuus L.) and Tithonia rotundifolia (Mill.) Blake. Helia 28: 61-68.

Robles, S. R. 1986. Producción de oleaginosas y textiles. Ed. Limusa S.A. México, D.F. pp. 431-436.

Schilling, E. E. y C. B. Heiser. 1981. Infrageneric classification of Helianthus (Compositae). Taxon 30: 393-403.

Upfold, S. J. y J. Van Staden. 1990. The germination characteristics of Tithonia rotundifolia. Ann. Bot. 66: 57-62.

Vos, P., R. Hogers, M. Bleker, M. Reijans, T. Van de Lee, M. Hornes, A. Frijters, J. Pot, J. Peleman, M. Kuiper y M. Zabeau. 1995. AFLP: a new technique for DNA fingerprinting. Nucl. Acids Res. 23: 4407-4414. 